\title{
Energy-Efficient Data Acquisition in Wireless Sensor Networks through Spatial Correlation
}

\author{
Li Xun and Tang Shiqi \\ School of Mechatronics Engineering and Automation \\ National University of Defense Technology \\ Changsha, Hunan Province, China \\ \{lixun, tangshiqi\}@nudt.edu.cn
}

\author{
Geoff V Merrett and Neil M White \\ School of Electronics and Computer Science \\ University of Southampton \\ Southampton, UK \\ \{gvm,nmw\}@ecs.soton.ac.uk
}

\begin{abstract}
The application of Wireless Sensor Networks (WSNs) is restrained by their often-limited lifetime. A sensor node's lifetime is fundamentally linked to the volume of data that it senses, processes and reports. Spatial correlation between sensor nodes is an inherent phenomenon to WSNs, induced by redundant nodes which report duplicated information. In this paper, we report on the design of a distributed sampling scheme referred to as the 'Virtual Sampling Scheme (VSS) '. VSS is formed from two components: an algorithm for forming virtual clusters and a distributed sampling method. VSS primarily utilizes redundancy of sensor nodes to get only a subset to sense the environment at any one time. Sensor nodes that are not sensing the environment are in a low-power sleep state, thus conserving energy. Furthermore, VSS balances the energy consumption amongst nodes by using a round robin method.
\end{abstract}

Index Terms - Virtual cluster; wireless sensor networks; spatial correlation.

\section{INTRODUCTION}

Wireless Sensor Networks (WSNs) consist of multiple sensor nodes [1] deployed over an area for monitoring some phenomena or tracking targets. In target tracking WSNs, sensor nodes cooperate to acquire the signal of the spatially and temporally dynamic target. Generally, a sensor node is powered by a local battery which has a limited energy budget. There is a contradiction for a sensor node that it should have a sufficient lifetime to finish the application task, while recharging the battery can be difficult, costly and even impossible in a hostile environment. Although a sensor node can be powered by energy harvesting technology [2], the energy obtained from the external environment is dependent on the environmental conditions and is rarely continuous.

Energy conservation is an important research field for WSNs. Anastasi et al. classified the majority of energy conservation techniques into duty cycled and data-driven approaches [3]. Communication protocols, such as S-MAC [4], commonly adopt a duty cycled approach. When communication is not required, a sensor node switches into a low-power sleep state to reduce the energy consumption. Recent research has shown that some types of sensors can consume more energy than the communication process [5]. Data-driven approaches to conserve energy can eliminate unnecessary sensing by adjusting the sample frequency and reducing the number of sensor nodes which participate in sensing. Methods of energy conservation often aim to put more sensor nodes into a sleep state, but tracking applications usually require a certain number of sensor nodes to be activate in order to sufficiently sample the target signal.

Generally, sensor nodes are densely deployed. Therefore sensor data from adjacent nodes will be highly redundant. Consecutive samples from the same node are also likely to exhibit temporal correlation. Vuran et al. analyzed the spatial and temporal correlation of WSNs [6]. Reducing redundant data can conserve a node's energy. Redundant information is not useful to data fusion, but sensing, processing and communication of the information can consume extra unnecessary energy.

Eliminating the acquisition of redundant data will have a significant effect on nodes in a WSN. The energy consumed on data acquisition and on communication will be both reduced. Without redundant data, the data processing is expedited in the microcontroller and a lower data rate will be created. So the occupation of the wireless channel and the probability of inducing congestion will be lower. As a result, the lifetime of WSNs is prolonged, and the QoS is increased.

In this paper, we investigate the energy conservation and balance (i.e. the distribution of energy throughout the nodes in the network) of WSNs. We present a novel scheme, referred to as Virtual Sampling Scheme (VSS), to fulfill distributed data acquirement in WSNs. VSS is based on virtual clusters (VCs), which represent a set of adjacent nodes. We design an algorithm to form VCs, which derives the VCs by using a spatial correlation model before nodes start to sample the target signal. The algorithm operates without calculating a complex distortion function as required by other reported research. The computation required in our algorithm is also lighter. We present a round robin sampling scheme as the second part of VSS, which balances the energy consumption of each sensor node in a VC. Therefore VSS assures the accuracy in signal estimation. The VSS algorithm is simulated to evaluate the fusion accuracy and energy consumption.

\section{RELATED WORK}

In a WSN, multiple sensor nodes often sample a signal at the same time. The node to fuse the sensor data from nodes may get more consecutive sensor data in a fusion periods. Two different methods can be used for data fusion, as Figure 1 shows: 


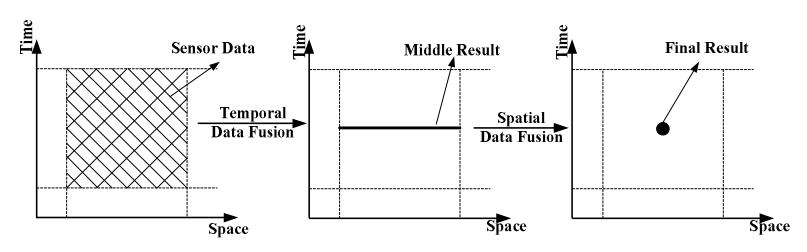

a: Temporal-spatial data fusion

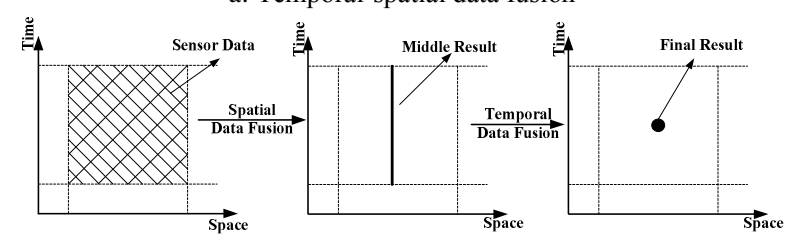

b: Spatial-temporal data fusion

Figure 1. Model of data fusion

The model showed in Figure 1a first takes the sensor data from each node and first fuses it in the temporal field. Then the middle results from all of nodes are fused to derive the final result. Figure 1b shows a similar model, but where the sensor data are first fused in the spatial field.

Whichever model is used, the key is how many nodes are selected, and how long is spent to obtain the final result. From the perspective of spatial correlation, it may be unnecessary for all nodes to participate in data acquisition. It has been shown that the accuracy of fusion depends on the number of nodes, the spatial correlation amongst nodes, and the spatial correlation between nodes and the source [6].

A number of papers have reported algorithms for data acquisition based on spatial correlation. Vuran and Akyildiz exploited spatial correlation on medium access control [7].They presented a theoretical framework under a distortion constraint. In the theoretical framework, they obtained a minimum node set, and every node in the set acted as representative node. To realize this framework, the CC-MAC protocol was developed which can regulate node to access medium. CC-MAC includes two parts, N-MAC and E-MAC, where E-MAC filters out the correlation in sensor records.

Willett et al. presented an adaptive sampling scheme, called 'Backcasting' [8]. The main idea of this scheme is that nodes deployed with sufficient density do not have to sense the same field simultaneously. A hierarchical approach of estimation and communication is proposed in their scheme. In the first phase, called preview, a small subset of the sensor nodes transmit their information to a fusion center, providing an initial estimation of the environment being sensed. In the second phase, called refinement, the fusion center activates additional sensors by 'Backcasting' an activation message.

Gedik et al. described an adaptive sampling approach to data collection, called ASAP [9]. ASAP consists of three main mechanisms. Firstly, nodes with close sensor readings are assigned to the same sub-cluster. Secondly, based on spatial correlation between nodes in one sub-cluster, one or more nodes are selected as samplers. The samplers sense the environment and transmit their data to the base station. Finally, adaptive data collection and model-based prediction are designed to minimize the number of messages used to extract data from the network.

In the above three papers, although the methods employed are different, all authors aim to get a subset of sensor nodes to participate in sampling process. Because of the existence of spatial correlation among adjacent nodes in a field, a subset of sensor nodes can represent the network to sense the environment. This paper presents an algorithm which utilizes spatial correlation, operates with a lower computational load (not requiring a complex distortion function), and balances the energy consumption of the network's nodes.

\section{NETWORK ARCHITECTURE AND SYSTEM MODEL}

\section{A. Networks architecture}

WSNs may have a flat or hierarchical architecture. A hierarchical WSN consists of clusters of nodes, where each cluster has a cluster head $(\mathrm{CH})$ responsible for communicating with other clusters. In this paper, a hierarchical WSN with $N$ sensor nodes is considered. Nodes $n_{1}, n_{2} \ldots n_{N}$ are stationary in a set $G$. Node $n_{i}(i=1,2, \cdots N)$ is at location $s_{i}\left(x_{i}, y_{i}\right)$. All sensor nodes are divided into $M$ clusters, every $\mathrm{CH}$ is resource-rich nodes which can perform data aggregation as presented in [10]. Let $C_{j}$ ( $j=1,2, \cdots M)$ denote the $j^{\text {th }}$ cluster. The $\mathrm{CH}$ of $C_{j}$ is $c h_{j}$ and the number of nodes (including $c h_{j}$ ) in $C_{j}$ is $M_{j}$, defined by equation (1).

$$
\begin{aligned}
& \sum_{j=1}^{M} M_{j}=N \\
& C_{j}=\left\{n_{i}, c h_{j} \mid n_{i} \in G, i=1,2 \cdots M_{j}-1\right\}
\end{aligned}
$$

\section{B. Spatial correlation model}

Spatial correlation between two nodes is decided by the distance between them. Berger et al. discussed four spatial correlation models [10]. The power exponential model is often used in applications, and defined in equation (2).

$$
K\left(d_{i j}\right)=e^{-d_{i j} / \theta_{1}}, \theta_{1}>0
$$

Where $d_{i j}$ is the distance between node $n_{i}$ and node $n_{j}$, calculated as equation (3).

$$
d_{i j}=\left\|s_{i}-s_{j}\right\|=\sqrt{\left(x_{i}-x_{j}\right)^{2}+\left(y_{i}-y_{j}\right)^{2}}
$$

At each observation point, the signals, from the source signal which is modeled as Gaussian random variables, are joint Gaussian random variable [6]. The noise is modeled as independent and identically distributed Gaussian random variables. Sensor data, combining signal with noise, can be obtained using equation (4).

$$
X_{i}=S_{i}+N_{i}
$$


Where $S_{i} \sim\left(0, \sigma_{S}^{2}\right)$ and $N_{i} \sim\left(0, \sigma_{N}^{2}\right)$.The covariance of sensor data of nodes $n_{i}$ and $n_{j}$ is given by equation (5).

$$
\begin{aligned}
& \operatorname{Cov}\left(X_{i}, X_{j}\right)=\operatorname{Cov}\left(S_{i}+N_{i}, S_{j}+N_{j}\right) \\
& =E\left(S_{i} S_{j}\right)+E\left(S_{i} N_{j}\right)+E\left(N_{i} S_{j}\right)+E\left(N_{i} N_{j}\right) \\
& =E\left(S_{i} S_{j}\right)=\sigma_{s}^{2} e^{-d_{i j} / \theta_{1}}
\end{aligned}
$$

If $d_{i j}$ is small enough, $\operatorname{Cov}\left(X_{i}, X_{j}\right)$ will be relatively large. $X_{i}$ and $X_{j}$ are highly redundant, nodes $n_{i}$ and $n_{j}$ do not need to join the sensing process together.

\section{VIRTUAL SAMPLING SCHEME}

\section{A. Definition of virtual cluster}

Definition: A node set (called $V C_{i j}$ ) which has $M_{i j}$ nodes is called a 'Virtual Cluster' of cluster $C_{i}$, if the following conditions are satisfied.

1) If $n_{k} \in V C_{i j}$,then: $n_{k} \in C_{i}, k=1,2, \cdots M_{i j}$

2) If $n_{k}, n_{l} \in V C_{i j}, k, l=1,2, \cdots M_{i j}$, then $\left\|s_{k}-s_{l}\right\| \leq d_{\text {min }}$

Where $d_{\min }$ is a distance threshold. A VC should have a head $(\mathrm{VCH})$ which implements a scheduling function. Assume that the VC number in $C_{i}$ is $K_{i}$, the $\mathrm{VCH}$ of $V C_{i j}$ is $v c h_{i j}$.

$$
\begin{aligned}
& V C_{i j}=\left\{n_{l}, v c h_{i j}, d_{\min } \mid n_{l} \in C_{i}, l=1 \cdots M_{i j}-1\right\} \\
& \sum_{j=1}^{K_{i}} M_{i j}=M_{i}, \quad i=1,2 \cdots M, j=1,2 \cdots K_{i}
\end{aligned}
$$

Figure 2 presents the relationship between cluster and VCs.

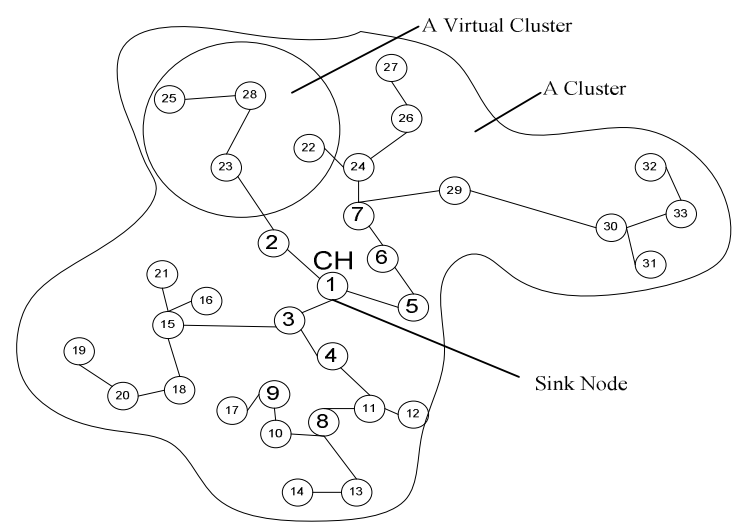

Figure 2. Cluster and virtual cluster

Note that a VC is meaningful when sensor nodes take part in sampling a signal. It does not affect the communication architecture of the network.

\section{B. Forming a virtual cluster}

A $\mathrm{VC}$ is a set of nodes with a $\mathrm{VCH}$ and a distance threshold. In order to form a $\mathrm{VC}$, we should set a $\mathrm{VCH}$, derive $d_{\min }$ and find nodes with the limitation $\left\|s_{k}-s_{l}\right\| \leq d_{\text {min }}$. A VC can be any shape in topology, and can be thought of as a circle whose center is $\mathrm{VCH}$ and radius is $d_{\text {min }} / 2$. All nodes belonging to the $\mathrm{VC}$ are in the circle and satisfy $\left\|s_{k}-s_{l}\right\| \leq d_{\text {min }}$. It is enough that only one sensor node in this $\mathrm{VC}$ performs sampling function at a time, other nodes can enter into sleep state.

Figure 3 shows an example of VCs, where nodes 1, 8, 15, 23, 26 and 30 are VCHs. The values $r$ and $d$ denote the communication radius of $\mathrm{CH}$ and circle radius of VCs, respectively.

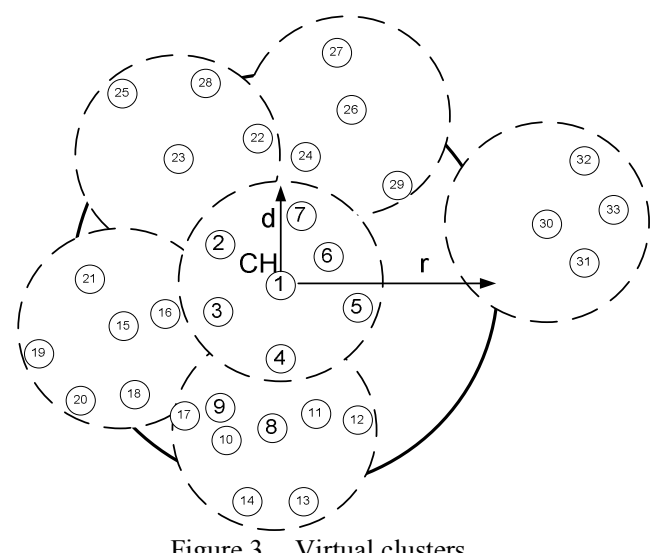

The process of forming VCs starts from the $\mathrm{CH}$. After the cluster is constructed, the process can begin. $\mathrm{CH}$ sets distance threshold and sends it to other nodes

Assumption 1: Every node in $G$ has an exclusive identification (ID) number. Some clustering algorithms are based on the node's ID number [11], so the assumption is easy to satisfy.

Assumption 2: Every node can calculate the distance to other nodes. Guoqiang Mao et al. presented some method about how to calculate the distance between nodes [12].

Assumption 3: The expected distance threshold is shorter than the communication radius of a node, as shown in Figure 3. The assumption can ensure the correlation amongst nodes is relatively high.

If a node in the cluster $C_{i}$, assume that it is $n_{k}$, wants to form a VC, it should broadcast a message with the expected distance value $d$ and its ID. Node $n_{k}$ will become a $\mathrm{VCH}$ after it broadcasts the message. Node $n_{l}$, which is still not in a $\mathrm{VC}$, will be at one of three possible states after $n_{k}$ broadcasts its message. The three possible states are as follows.

State1: $S 1 \rightarrow\left\|s_{k}-s_{l}\right\| \leq d$

State2: $S 2 \rightarrow\left\|s_{k}-s_{l}\right\| \leq r,\left\|s_{k}-s_{l}\right\|>d$ 


\section{State3: $S 3 \rightarrow\left\|s_{k}-s_{l}\right\|>r$}

If node $n_{l}$ receives the broadcast message, it calculates the distance $\left\|s_{k}-s_{l}\right\|$ and compares it with $d$. Node $n_{l}$ will join the $\mathrm{VC}$ if it is in state $\mathrm{S} 1$ after comparison. It will reply to $n_{k}$ with its ID. If node $n_{l}$ is in state S2, it will set a timer $\mathrm{T}_{1}$ with random time once it receives the broadcast message. Before $\mathrm{T}_{1}$ expires, $n_{l}$ should keep listening to a new message. If it can change to state $\mathrm{S} 1$, it will send a reply message and join the $\mathrm{VC}$ that the $\mathrm{VCH}$ is the node which sends the broadcast message. When $\mathrm{T}_{1}$ expires, $n_{l}$ sends a broadcast message to claim a new VC. Node $n_{l}$ will be a $\mathrm{VCH}$.

Initially, all nodes are in state $S 3$, every node sets a timer $T_{2}$ with random time, which should be longer than $T_{1}$. Before $T_{2}$ expires, a node should listen to a broadcast message and change state to $\mathrm{S} 1$ or $\mathrm{S} 2$. When timer $\mathrm{T}_{2}$ of a node expires, the node can not receive a broadcast message, and it does not know value $d$. E.g., In Figure 2, although node 30 is close to node 29 , but node 29 can not broadcast a claiming message after it joins a VC, so node 30 can not receive any broadcast message. Once $T_{2}$ expires, node 30 broadcasts an enquiring message with its ID. Node 29 will receive the enquiring message and send a reply with value $d$. After receiving the value $d$, node 30 broadcasts a message to claim a new VC. Figure 4 presents the states relationship. In Figure 4, state S5 means that the node has been a member of one VC. S4 is a middle state. In S4, a node will send a broadcast message to become a $\mathrm{VCH}$ after it receives a reply for its enquiry.

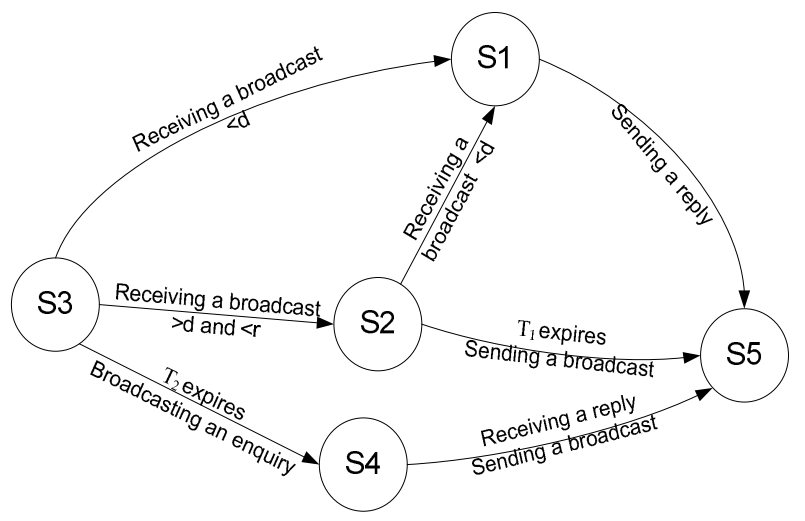

Figure 4. States relationship in one node

When a VC has been formed, the VCH should maintain a ID table. Every node in a VC will receive some parameters from their $\mathrm{VCH}$, and this $\mathrm{VCH}$ should report its ID to the $\mathrm{CH}$. Each $\mathrm{VCH}$ will receive some parameters from a $\mathrm{CH}$ and sends these parameters to each node, such as the sample frequency.

There is no central node to control the forming process, it is distributed. The $\mathrm{CH}$ will be the first node to broadcast message. Note that a node in state S5 will not reply to any broadcast message from other nodes to claim a new VC. Figure 5 describes the algorithm running at a node.

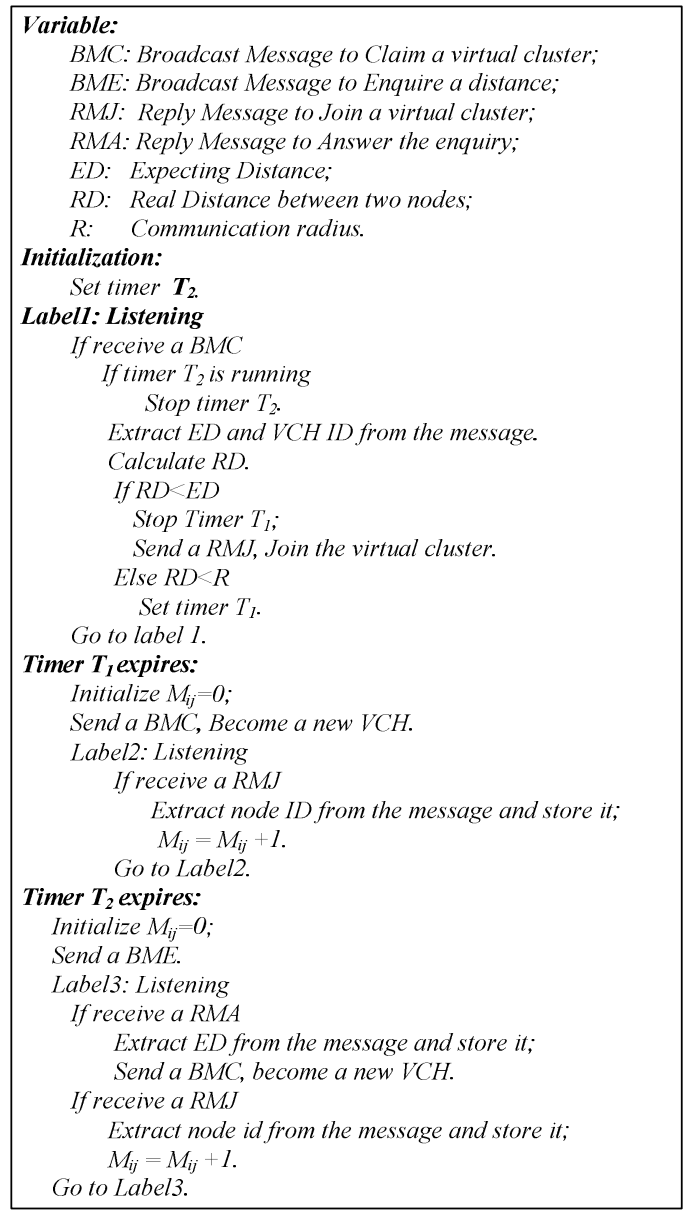

Figure 5. Forming algorithm

\section{Distributed round robin sampling in a virtual cluster}

In a VC, all nodes are equal. Every node can perform sampling function as the representative of the VC. We can set a node to sample the signal while other nodes can work in sleep state. But sampling node will consume its energy more quickly than others. We adopt a distributed round robin sampling scheme to balance energy amongst sensor nodes.

While forming a VC, the $\mathrm{VCH}$ receives the reply from other nodes with their ID, and knows the total number of nodes and their ID. Assume that the ID table is $\left\{i d_{1}, i d_{2}, \cdots i d_{M_{i j}}\right\}$ of $V C_{i j}$, ordered from small to large. Every ID has an index in the table, $\operatorname{Index}\left(i d_{k}\right)=k$. The round robin scheme requires all nodes in the $\mathrm{VC}$ to synchronize with $v c h_{i j}$. Because there are only limited nodes in a $\mathrm{VC}$, the reference broadcast synchronization method [13] can be used. The distributed sampling process has two steps: allocation and start, both steps are scheduled by $v c h_{i j}$. 
Allocation: $v c h_{i j}$ allocates sample time for all nodes. The round robin period is $T=M_{i j} / f_{\min }$. The $i d_{k}$ node samples the source signal at time:

$$
t_{0}+\frac{k}{f_{\text {min }}}+T \times m, \quad(m=1,2, \cdots)
$$

Where $t_{0}$ is the start time to sample, $f_{\text {min }}$ is the expected sample rate. In this step, $v c h_{i j}$ sends parameters $T, k$ and $f_{\text {min }}$ to every node in the same VC.

Start: This step starts the sampling process. $v c h_{i j}$ sends a start command with starting time $t_{0}$, every node start to sample according to equation (7).

Figure 6 shows the sampling and sleeping period of nodes.

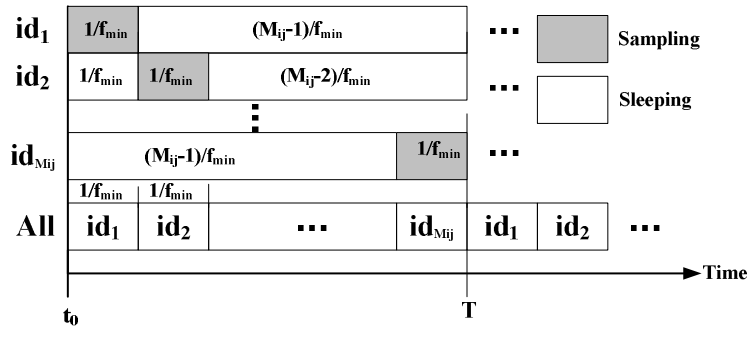

Figure 6. Sampling schedeme

We use the same method discussed in [6] to fuse the data and analyze the tracking error. Minimum mean square error estimation is used to estimate the event $S_{i}$ signal. The estimation, $Z_{i}$, is:

$$
Z_{i}=\frac{E\left(S_{i} X_{i}\right)}{E\left(X_{i}^{2}\right)} X_{i}=\frac{\sigma_{S}^{2}}{\sigma_{S}^{2}+\sigma_{N}^{2}} X_{i}=\frac{\sigma_{S}^{2}}{\sigma_{S}^{2}+\sigma_{N}^{2}}\left(S_{i}+N_{i}\right)
$$

The estimation of $S$, also the fusion result, is:

$$
\bar{S}=\frac{1}{K} \sum_{i=1}^{K} Z_{i}=\frac{\sigma_{S}^{2}}{K\left(\sigma_{S}^{2}+\sigma_{N}^{2}\right)} \sum_{i=1}^{K} X_{i}=\frac{\sigma_{S}^{2}}{K\left(\sigma_{S}^{2}+\sigma_{N}^{2}\right)} \sum_{i=1}^{K}\left(S_{i}+N_{i}\right)
$$

The distortion between the source and the fusion result is:

$$
\begin{aligned}
D=E\left[(S-\bar{S})^{2}\right]= & \sigma_{S}^{2}+\frac{\sigma_{S}^{4}}{K\left(\sigma_{S}^{2}+\sigma_{N}^{2}\right)} \\
& -\frac{2 \sigma_{S}^{4}}{K\left(\sigma_{S}^{2}+\sigma_{N}^{2}\right)} \sum_{i=1}^{K} \rho_{s, i}+\frac{\sigma_{S}^{6}}{K^{2}\left(\sigma_{S}^{2}+\sigma_{N}^{2}\right)^{2}} \sum_{i=1}^{K} \sum_{j \neq i} \rho_{i, j}
\end{aligned}
$$

Where $\rho_{s, i}$ is the spatial correlation coefficient between source and nodes $n_{i}, \rho_{i, j}$ is the spatial correlation coefficient between nodes $n_{i}$ and $n_{j}$.

\section{Analysis of energy conservation}

In every $T$ interval, a node is active for $1 / f_{\min }$ time to sample the signal. Assume that the consuming power of sensing unit of a sensor node in active mode and sleep state is $P_{A}$ and $P_{S}$, respectively. The energy consumption of a node is:

$$
E_{N}=P_{A} \times \frac{1}{f_{\min }}+P_{s} \times\left(T-\frac{1}{f_{\min }}\right)=\frac{1}{f_{\min }} \times\left[P_{A}+P_{s} \times\left(M_{i j}-1\right)\right]
$$

If the round robin sampling scheme is not adopted, a node should be active in the whole $T$ interval, the conservation of energy of a node is:

$$
1-\frac{E_{N}}{P_{A} \times T}=\left(1-\frac{1}{M_{i j}}\right) \times\left(1-\frac{P_{S}}{P_{A}}\right) \approx 1-\frac{1}{M_{i j}}
$$

Only a node is active in a VC within $1 / f_{\min }$ interval, the VC will consume energy:

$$
E_{V}=P_{A} \times \frac{1}{f_{\text {min }}}+P_{S} \times\left(M_{i j}-1\right) \times \frac{1}{f_{\text {min }}}
$$

If the round robin scheme is used, the ratio of which a $\mathrm{VC}$ conserves energy is:

$$
1-\frac{E_{V}}{P_{A} \times M_{i j} \times \frac{1}{f_{\min }}}=\left(1-\frac{1}{M_{i j}}\right) \times\left(1-\frac{P_{S}}{P_{A}}\right) \approx 1-\frac{1}{M_{i j}}
$$

From equations (12) and (14), we can conclude that a node conserves energy as the same level as the $\mathrm{VC}$ which the node belongs to. The ratio of energy conservation is only related with the number of nodes in the VC. The number of nodes is larger, the energy conservation is more.

\section{Simulation ReSUlts}

The simulation environment has 100 nodes and covers a $50 \times 50 \mathrm{~m}^{2}$ area. Each node is randomly deployed in this area. The radius of a $\mathrm{VC}$ is set as $10 \mathrm{~m}$. The result of simulation shows fifteen VCs are formed if the algorithm illustrated in Figure 5 is used. Figure 7 illustrates the fifteen VCHs and, as an illustration, the location and distribution of VCs one to five.

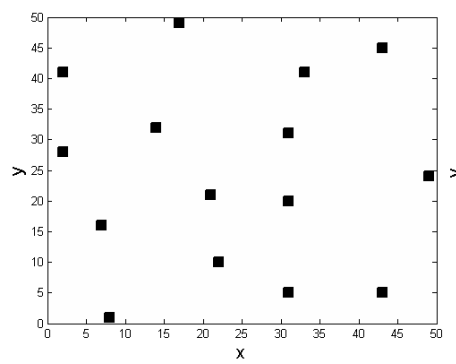

a: $\mathrm{VCH}$

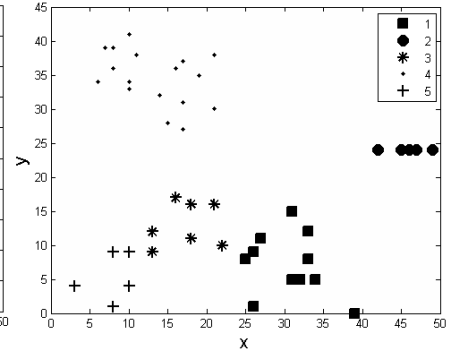

b: $1^{\text {th }}-5^{\text {th }} \mathrm{VCs}$
Figure 7. Distribution of $\mathrm{VCH}$ and $\mathrm{VCs}$

There is only one source signal in the simulation. The signal is a Gaussian random variable with expectation and variance of $(0,1)$. The noises at each observed location are joint Gaussian random variables with expectations and variances of $(0,0.01)$. By using the fusion method given in equation (9), Figure 8 shows the fusion result and the variance of the fusion result at a fusion node. The number of samples is 100 . 


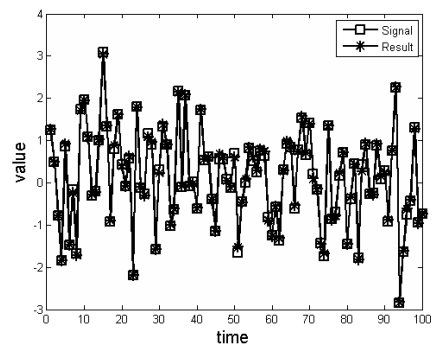

a: Fusion result

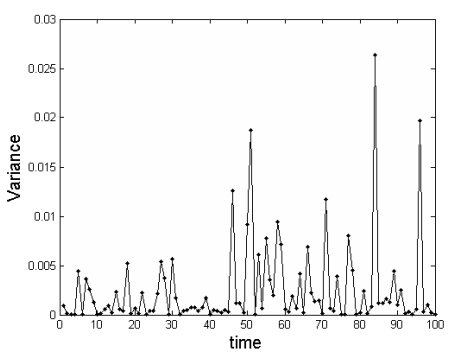

b: Variance
Figure 8. Fusion result and variance

At each sample time, each VC has a node to sample the signal. Hence, fifteen sensor data are fused in every sample period. Because the number of nodes in each $\mathrm{VC}$ is not equal, the combination of nodes to sample the signal is different. According to equation (10), although the number of nodes which join the fusion process is the same, the distortion is different. This is illustrated in Figure $8 \mathrm{~b}$. A few points in Figure $8 \mathrm{~b}$ show a greater variance, but in most cases, although only a subset of the nodes take part in the sampling process at any one time, the fusion result is improved.

In Figure 9, the energy conservation of the $\mathrm{VCs}$ is shown. The ratio of energy conservation of a $\mathrm{VC}$ is calculated as equation (14) shows. In order to compare different VCs, we set the radius of the $\mathrm{VC}$ as $5 \mathrm{~m}$ and $10 \mathrm{~m}$, respectively.

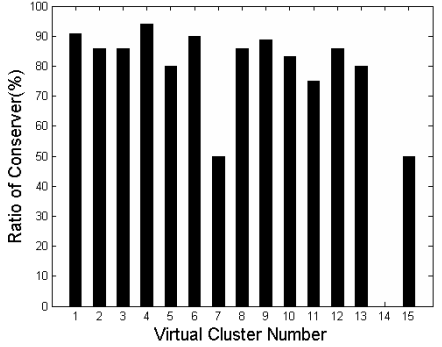

a: Radius $=10 \mathrm{~m}$

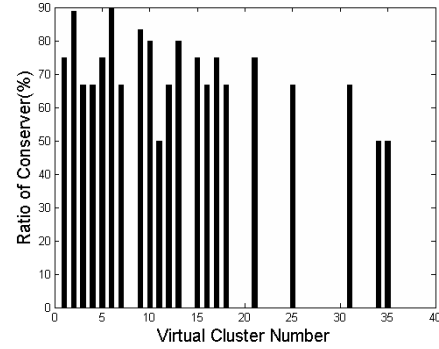

b: Radius $=5 \mathrm{~m}$
Figure 9. Ratio of energy conservation

The ratio of energy conservation is proportional to the number of nodes in a VC. As the radius of a VC increases, the number of nodes inside the $\mathrm{VC}$ also increases, and hence permits better energy conservation (as each node spends more of its time in a sleep state).Figure 9a shows the largest ratio is $95 \%$, while the largest ratio is $90 \%$ in Figure 9b. Some VCs, such as VC14 in Figure 9a, has only one node, the node will continue to sample the signal and can not conserve energy according to VSS. Because the spatial correlation model is related to the radius, careful consideration should be taken in the selection of the radius. A larger radius can give better energy conservation, though may result in worse fusion. As proved in section III, each node in the VC consumes the same amount of energy. Therefore, Figure 9 is also showing the energy conservation of the individual nodes.

\section{CONCLUSION}

Based on spatial correlation among adjacent sensor nodes, this paper presents a distributed virtual sampling scheme, referred to as VSS. VSS is a novel method to acquire data in a
WSN. In order to realize VSS, we design a round robin sampling scheme and an algorithm to form the VCs. Through analysis and simulation, it is proved that VSS can conserve node's energy while also providing a better fusion result. By VSS, each node in a VC consumes the same amount of energy, hence balancing energy consumption.

VSS can reduce redundant sensor data to conserve energy, while retaining the meaningful information. However, if a VC has few nodes, the nodes in this VC will consume energy at a faster rate. This raises a significant challenge, and we are currently investigating refinements to VSS to balance the energy between different VCs. Furthermore, while VSS should ensure the accuracy of the fusion result, our simulation results have shown that, in a small number of cases, VSS can give a worse result. By combining the spatial correlation with the number of nodes, we aim to find the optimal node set to get a better fusion result while keeping a high efficiency of energy conservation.

\section{REFERENCES}

[1] I.F.Akyildiz,W.Su,Y.Sankarasubramaniam and E.Cayirci, "Wireless sensor networks: a survey," Computer Networks,Vol.38,pp. 393-422, 2002.

[2] Merrett, G. V., Harris, N. R., Al-Hashimi, B. M. and White, N. M. Energy Managed Reporting for Wireless Sensor Networks. Sensors and Actuators A: Physical, Vol.142, pp. 379-389, 2008.

[3] Anastasi G.,Francesco M.D.,Conti M. and Passarella A., "Energy conservation in wireless sensor networks: A survey," Ad Hoc Networks, Vol. 7, pp.537-568,2009.

[4] W. Ye, J. Heidemann and D. Estrin, "Medium Access Control With Coordinated Adaptive Sleeping for Wireless Sensor Networks," IEEE/ACM Transactions on Networking, Vol.12, pp.493-506,2004.

[5] V. Raghunathan, S. Ganeriwal and M. Srivastava, "Emerging Techniques for Long Lived Wireless Sensor Networks," IEEE Communications Magazine, pp.108-114,2006.

[6] M.C.Vuran,O.B.Akan,andI.F.Akyildiz,"Spatio-Temporal Correlation: Theory and Applications for Wireless Sensor Networks," Computer Networks Journal (Elsevier), Vol. 45, no. 3, pp. 245-261, June 2004.

[7] Mehmet C. Vuran and Ian F. Akyildiz, "Spatial Correlation-Based Collaborative Medium Access Control in Wireless Sensor Networks," IEEE/ACM TRANSACTIONS ON NETWORKING, Vol. 14, No. 2, pp. 316-329, April 2006.

[8] R.Willett, A.Martin and R.Nowak, "Backcasting: adaptive sampling for sensor networks," Proc. of the Third International Symposium on Information Processing in Sensor Networks IPSN 2004), pp. 124-133, April 2004.

[9] Gedik.B., Ling Liu and P.S. YU, "ASAP: An Adaptive Sampling Approach to Data Collection in Sensor Networks," IEEE Transactions on Parallel and Distributed Systems, vol. 18, pp. 1766 - 1783,December 2007.

[10] J.O. Berger, V. de Oliviera and B. Sanso, "Objective bayesian analysis of spatially correlated data," Journal of the American Statistical Association, Vol.96, pp.1361-1374,2001.

[11] Ameer Ahmed Abbasi and Mohamed Younis, "A survey on clustering algorithms for wireless sensor networks," Computer Communications, Vol.30, pp. 2826-2841, 2007.

[12] Guoqiang Mao, Barıs_Fidan and Brian D.O. Anderson, "Wireless sensor network localization techniques,"Computer Networks,Vol.51, pp. 2529 2553, 2007.

[13] J.Elson,L.Girod and D.Estrin, "Fine-grained network time synchronization using reference broadcasts," In Proceedings of Fifth Symposium on Operating Systems Design and Implementation, Vol.36, pp.147-163, 2002. 